\title{
Eficiência dos institutos de pesquisa agropecuária do estado de São Paulo: avaliação de indicadores do PPA 2004-2013
}

José Roberto Vicente ${ }^{1}$ Alexandre Meloni Vicente ${ }^{2}$

1 Doutor em Economia. Pesquisador do Instituto de Economia Agrícola (IEA) e assessor técnico do gabinete do coordenador da Agência Paulista de Tecnologia dos Agronegócios (APTA).

2 Doutorando pelo Departamento de Política Científica e Tecnológica da Universidade Estadual de Campinas (Unicamp). 


\section{Resumo}

MÉTOdOS OQUE AUXILIEM NA AVALIAÇÃO DA EFICIÊNCIA DE INSTITUIÇÕES DEDICADAS À GERAÇÃO DE INOVAÇÕES SÃO IMPORTANTES PARA A SUSTENTABILIDADE ECONÔMICA E PARA A TRANSPARÊNCIA NA GESTÃO DOS DISPÊNDIOS PÚBLICOS. O SISTEMA ESTADUAL DE PESOQUISA AGROPECUÁRIA do estado de SÃo Paulo, coordenado pela Agência Paulista de TECNOLOGIA doS AgRONEGócios (APTA), É FORMADO PELOS INSTITUTOS DE PESOUISA AGRONÔMICO (IAC), BIOLÓGICO, DE ECONOMIA AGRÍCOLA (IEA), PESCA, TECNOLOGIA DE ALIMENTOS (ITAL) E ZOOTECNIA (IZ), ALÉM DE 14POLOS REGIONAIS (APTA REGIONAL). 0 ESTUDO COMPAROU OS NÍVEIS DE EFICIÊNCIA DESSAS INSTITUIÇÕES NO PERÍODO DE 2004 A 2013, UTILIZANDO ANÁLISE ENVOLTÓRIA DE DADOS (DEA). OS PRODUTOS FORAM INDICADORES DE GERAÇÃO E TRANSFERÊNCIA DE CONHECIMENTO E CAPTAÇÃO DE RECURSOS, E OS INSUMOS FORAM OS ORÇAMENTOS ANUAIS. OS RESULTADOS OBTIDOS MOSTRARAM ASSOCIAÇÃO ENTRE OS ÍNDICES DE EFICIÊNCIA E AS DIFERENTES MISSÕES INSTITUCIONAIS. ALGUMAS UNIDADES APARENTARAM SER MAIS EFICIENTES NA GERAÇÃO, E OUTRAS, NA TRANSFERÊNCIA DO CONHECIMENTO. AS UNIDADES OPERARAM NAS FRONTEIRAS DE EFICIÊNCIA DA GERAÇÃO OU DA TRANSFERÊNCIA DE CONHECIMENTO EM PELO MENOS UM DOS ANOS DO PERÍODO ANALISADO.

\section{Abstract}

METHODS THAT HELP IN ASSESSING THE EFFICIENCY OF INSTITUTIONS DEDICATED TO THE GENERATION OF INNOVATIONS ARE IMPORTANT FOR ECONOMIC SUSTAINABILITY AND TRANSPARENCY IN THE MANAGEMENT OF PUBLIC EXPENDITURES. THE AGRICULTURAL RESEARCH SYSTEM OF THE STATE OF SÃO PAULO, COORDINATED by the AgênCia Paulista de TeCnologia dos AGRONEGÓCIOS (APTA), IS FORMED BY THE RESEARCH INSTITUTES: AGRONÔMICO (IAC), BIOLÓGICO (IB), Economia Agrícola (IEA), Pesca, TeCnologia de ALIMENTOS (ITAL) E ZOOTECNIA (IZ), PLUS FOURTEEN Regional Poles (APTA Regional). The Study COMPARED THE EFFICIENCY LEVELS OF THESE INSTITUTIONS BETWEEN 2004-2013, USING DATA ENVELOPMENT ANALYSIS (DEA). THE PRODUCTS WERE INDICATORS OF GENERATION AND TRANSFER OF KNOWLEDGE, AND FUNDRAISING; INPUTS WERE THE ANNUAL BUDGETS. THE RESULTS SHOWED AN ASSOCIATION BETWEEN THE EFFICIENCY INDICES AND THE DIFFERENT INSTITUTIONAL MISSIONS. SOME UNITS APPEAR TO BE MORE EFFICIENT IN THE GENERATION AND OTHERS IN KNOWLEDGE TRANSFER. UNITS OPERATED ON THE EFFICIENCY FRONTIERS OF GENERATION OR KNOWLEDGE TRANSFER IN AT LEAST ONE OF THE YEARS ANALYZED.

\section{PALAVRAS-CHAVE:}

Pesquisa agrícola; Eficiência; Fronteira não paramétrica.

Revista Brasileira de Monitoramento e Avaliação | Número 9 | Janeiro-Junho de 2015

Eficiência dos institutos de pesquisa agropecuária do estado de São Paulo: avaliação de indicadores do PPA 2004-2013 


\section{Introdução}

O sistema de geração de tecnologia para a agricultura brasileira é, em grande parte, financiado por recursos públicos, principalmente através de instituições públicas de pesquisa. A avaliação do desempenho dessas organizações é importante não apenas como subsídio ao planejamento e gestão, mas também para responder à sociedade sobre a alocação dos recursos públicos.

Com o fortalecimento da doutrina neoliberal, especialmente a partir do Consenso de Washington, no final da década de 1980, robusteceu-se a defesa de um maior distanciamento do setor público com relação à responsabilidade de geração e difusão da pesquisa agrícola (VICENTE, 1998). O processo de diminuição de recursos para pesquisa- observado desde o início daquela década- levou a uma involução de sistemas oficiais de pesquisa em países em desenvolvimento, comprometendo a eficácia de sua atuação (VICENTE, 2002).

Segundo Sachs (2008), as descobertas científicas, das quais dependem as tecnologias sustentáveis, são um bem público que não pode ser fornecido pelas forças de mercado. Isso porque o conhecimento científico é um bem intangível, que pode ser usado por uma pessoa sem diminuir sua disponibilidade para as demais, e funciona de forma mais efetiva quando é amplamente compartilhado, oferecendo, consequentemente, uma base comum para a compreensão, a ação e o desenvolvimento de sistemas tecnológicos. Por conseguinte, continua o autor, o funcionamento da ciência depende, em parte, da publicação rápida e gratuita das descobertas pela comunidade científica: "Os cientistas não guardam diretamente para si muitos dos benefícios econômicos de suas descobertas (se é que o fazem), e nem devem, se esse conhecimento tem um impacto de utilidade máximo" (SACHS, 2008). E, para que essas descobertas permaneçam disponíveis ao público, meios não relacionados ao mercado devem ser usados para apoiar o investimento financeiro de recursos na descoberta científica. Mesmo sem ser completamente compreendida pelos ideólogos do livre mercado, "[...] a necessidade de recursos públicos e filantrópicos é amplamente reconhecida nos Estados Unidos [...] É por isso que os EUA, o paradigma do livre mercado, gastam mais de cem bilhões de dólares por ano dos fundos orçamentários federais com pesquisa e desenvolvimento" (SACHS, 2008).

Torna-se, portanto, evidente a importância das instituições públicas de pesquisa. E sua existência depende cada vez mais de mecanismos eficientes de gestão e de demonstrações efetivas dos resultados e impactos de suas atividades.

O Governo do Estado de São Paulo mantém vinculada à Secretaria de Agricultura e Abastecimento e coordenada pela Agência Paulista de Tecnologia dos Agronegócios (APTA) - uma tradicional estrutura de pesquisa agropecuária, composta pelo Instituto Agronômico (IAC), Instituto Biológico (IB), Instituto de Economia Agrícola (IEA), Instituto de Pesca, Instituto de Tecnologia de Alimentos (Ital), Instituto de Zootecnia (IZ) e pelo Departamento de Descentralização 
do Desenvolvimento (APTA Regional), este último com 14polos regionais de desenvolvimento tecnológico dos agronegócios (PRDTA).

A Constituição Federal de 1988 define, no Artigo 155, os três instrumentos do modelo orçamentário brasileiro: o Plano Plurianual (PPA), a Lei de Diretrizes Orçamentárias (LDO) e a Lei Orçamentária Anual (LOA). A LDO, que identifica no PPA as ações que receberão prioridade no exercício seguinte, é o elo entre o PPA- o plano de médioprazo do governo- e a LOA, que é o instrumento que viabiliza a execução do plano de trabalho do exercício em questão. O planejamento expresso no Plano Plurianual assume a forma de grande moldura legal e institucional para a ação nacional, bem como para a formulação dos planos regionais e setoriais. Um argumento forte em relação à importância que os constituintes deram ao planejamento no Brasil está expresso no $\S 1^{\circ}$ do Inciso XI do Art. 167 da Constituição Federal: "Nenhum investimento cuja execução ultrapasse um exercício financeiro poderá ser iniciado sem prévia inclusão no plano plurianual, ou sem lei que autorize a inclusão, sob pena de crime de responsabilidade" (GONTIJO, 2014).

Os programas e as ações (atividades e projetos) explicitados no PPA, assim como seus respectivos indicadores de realização das metas previstas, são disponibilizados para consultas públicas pelas diferentes esferas de governo e podem ser utilizados por distintos setores da sociedade para verificar a adequação do uso dos recursos disponíveis.

A partir do PPA 2004-2007, as ações (projetos e atividades) executadas pela APTA concentraram-se no Programa 1301, então chamado de Inovação Tecnológica para Competitividade dos Agronegócios (Agroinova São Paulo). Nos PPAs 2008-2011 e 2012-2015, o Programa 1301 foi denominado de Geração e Transferência de Conhecimento e Tecnologias para o Agronegócio.

A análise de indicadores de geração e transferência de conhecimento necessita de métodos adequados para possibilitar interpretações sobre o desempenho institucional. A análise de encapsulamento de dados (DEA), um método não paramétrico consagrado de construção de medidas de eficiência através de inputs e outputs, possibilita mensurar o desempenho relativo de uma unidade produtiva, apontando pontos fortes e fracos, e permite indicar o máximo de produção possível com o nível atual de uso de insumos, ou a realocação de insumos para uma melhor eficiência.

O objetivo deste estudo foi mensurar a eficiência relativa das instituições de pesquisa coordenadas pela Agência Paulista de Tecnologia dos Agronegócios (APTA), através da análise de uma década de indicadores do PPA, no período de 2004 a 2013.

Revista Brasileira de Monitoramento e Avaliação | Número 9 | Janeiro-Junho de 2015 


\section{Metodologia}

A análise envoltória de dados (DEA) é uma alternativa de construção de fronteiras de melhor prática, sem necessidade de especificação da tecnologia de produção, proposta por Charnes, Cooper e Rhodes (1978). A eficiência de uma determinada "unidade tomadora de decisões (DMU)" é medida em relação a todas as outras unidades, com a restrição simples de que todas elas se encontram abaixo da fronteira eficiente ou, no máximo, sobre ela (SEIFORD e THRALL, 1990).

Mais especificamente, trata-se de uma metodologia de programação linear que utiliza dados de quantidades de produtos(y) e de insumos ( $x$ ) na construção de uma superfície linear compreensível. A superfície de fronteira é obtida pela resolução de uma sequência de problemas de programação linear, um para cada observação (DMU) da amostra (RAO e COELLI, 1999). O grau de ineficiência de cada DMU é obtido pela distância de cada ponto até a fronteira. No presente estudo, foi utilizado um modelo DEA produto-orientado, que define a fronteira procurando o nível máximo de produto, mantendo inalterado o uso dos insumos de cada observação.

Utilizando o teorema da dualidade, o problema de programação matemática, considerando-se retornos constantes à escala (modelo CCR), pode ser representado por (COELLI, 1996): $\min \theta, \lambda \theta$, sujeito às restrições: $-y i+Y \lambda \geq 0$; $\theta x i-X \lambda \geq 0 ; \lambda \geq 0$, onde $Y$ é uma matriz $(M \times N)$ representativa de $M$ produtos para N DMUs, $X$ é uma matriz de $K$ insumos ( $K x N), \theta \leq 1$ é um escalar, representativo do grau de eficiência técnica (ET) da i-ésima DMU, e $\lambda$ é um vetor (N×1) de constantes. Um valor de $\theta=1$ indica um ponto sobre a fronteira, ou seja, uma DMU eficiente de acordo com a definição de Farrell (1957). No caso de uma DMU eficiente, todos os valores de $\lambda$ serão iguais a zero; para as DMUs ineficientes, os valores de $\lambda$ são os pesos utilizados na combinação de DMUs eficientes que projetam cada DMU ineficiente sobre a fronteira. Esse problema de programação linear tem que ser resolvido $\mathrm{N}$ vezes, uma para cada observação da amostra.

A assunção de retornos constantes à escala pode ser relaxada impondo-se uma nova restrição para permitir retornos variáveis (ou BCC, BANKER, CHARNES e COOPER, 1984): $N 1$ ' $\lambda \geq 0$, onde $\mathrm{N} 1$ é um vetor $\mathrm{N} \times 1$ com todos os elementos iguais a 1 .

No modelo de retornos variáveis à escala, a intersecção de planos forma uma superfície convexa que envelopa os dados mais estreitamente do que a superfície cônica do modelo de retornos constantes. Dessa forma, as medidas de eficiência técnica provenientes do BCC são iguais ou maiores do que as do CCR (COELLI et al., 2005).

O software utilizado para a construção das fronteiras foi o DEA-Solver LV 3.0 (COOPER, SEIFORD e TONE, 2007).

A nova dinâmica de interação das variáveis que envolvem as atividades de pesquisas, recursos financeiros, resultados, geração e transferência do conhecimento foi discutida por Stokes (2005) a partir dos chamados mo- 
delos de inovação. As atividades de pesquisa inspiradas pelo uso ou pelo conhecimento podem ser enquadradas como: quadrante de Bohr, em que estão situadas aquelas inspiradas apenas pelo entendimento ou pesquisa básica; as pesquisas inspiradas pelo uso e entendimento, que estariam no quadrante de Pasteur; e, por fim, o quadrante de Edison, que enquadra as pesquisas voltadas apenas para a aplicação (VEIGA FILHO; VICENTE; MARTINS, 2011). Essas categorias foram aplicadas por Rio (2009) sobre as medidas de eficiência na geração e na transferência de conhecimento estimadas por Vicente e Martins (2006) para os institutos de pesquisa da Agência Paulista de Tecnologia dos Agronegócios (APTA), e por Veiga Filho; Vicente; Martins (2011) para os PRDTAs da APTA. Essa tentativa de classificação e ilustração das atividades de pesquisa foi também utilizada neste estudo, como forma de subsidiar a avaliação e o planejamento de ações estratégicas.

\subsection{VARIÁVEIS, FONTES DE DADOS E ESPECIFICAÇÃO DOS MODELOS}

Os indicadores de produção institucionais foram divididos em dois grupos: indicadores de geração e indicadores de transferência de conhecimento e serviços.

Como indicador de geração de conhecimento consideraram-se as pesquisas em andamento nas unidades da APTA, cadastradas no Sistema de Informações Gerenciais do Agronegócio (SIGA), e trimestralmente inseridas no
Sistema de Monitoramento de Programas e Ações do PPA (Simpa), mantido pela Secretaria de Planejamento e Desenvolvimento Regional. Esse é o indicador de metas da Ação 5925 (Geração de Conhecimento e Tecnologias para o Agronegócio), do Programa 1301.

Mesmo do ponto de vista quantitativo, esse pode ser considerado um indicador limitado dos resultados de pesquisas, mas a dinâmica do PPA, que exige dados das metas realizadas durante e logo ao final do ano em curso, impede que sejam consideradas, por exemplo, as publicações resultantes desses estudos em andamento, normalmente concretizadas após vários meses ou mesmo anos.

No grupo de transferência de conhecimento (e prestação de serviços), foram utilizados indicadores lançados mensalmente no Simpa/PPA: o número de análises laboratoriais realizado, indicador de metas da Ação 4872 (Análises Laboratoriais para a Qualidade e Segurança Alimentar); e os atendimentos técnicos diretos, as pessoas treinadas, e os acessos aos sites institucionais das unidades da APTA, marcos de monitoramento da Ação 4891 (Transferência de Conhecimento para o Agronegócio).

Em seguida, para obter um índice único representativo da transferência de conhecimento, adaptou-se o esquema utilizado originalmente por Khan e Silva (2005):IG $=\frac{1}{n} \sum_{n}^{\mathrm{i}=1} \frac{\mathrm{E}_{\mathrm{ij}}}{\mathrm{E}_{\max \mathrm{i}}}$ onde IG =índice de geração de conhecimento; $E_{i j}$ = escore do i-ésimo indicador para a j-ésima

Revista Brasileira de Monitoramento e Avaliação | Número 9 | Janeiro-Junho de 2015 
unidade; $E_{\text {maxx.j }}=$ escore máximo do i-ésimo indicador; $i=1, \ldots, n$, número de indicadores. Esse índice pode assumir valor entre 0 e 1 ou,se multiplicado por 100, representar um percentual. Não foram atribuídos pesos distintos aos indicadores agregados por essa fórmula.

Como insumos, foram considerados: despesas com pessoal (pesquisadores, técnicos e funcionários administrativos) e custeio (utilidade pública, como água, energia elétrica e telefones; contratos e despesas operacionais, tais como segurança, limpeza, e outros serviços diversos; demais insumos, principalmente combustíveis, fertilizantes, defensivos, rações e medicamentos para animais, materiais para escritório e diárias), somando-se ao custeio os investimentos realizados (obras, instalações, benfeitorias, veículos, máquinas e equipamentos diversos). No caso dos investimentos, adotou-se a seguinte ponderação: 0,15 para os efetuados no ano em curso $\left(\mathrm{t}_{0}\right), 0,35$ para os efetuados no ano anterior $\left(t_{-}{ }_{1}\right), 0,5$ para os efetuados no ano retrasado $\left(t_{2}\right)$. A esses investimentos foram somados ainda os efetuados em anos precedentes com os pesos: 0,75 para os de $t_{-}$; 0,5 para os de $\mathrm{t}_{-4}$; $\mathrm{e}$ 0,25 para os de t- ${ }_{5}$. Considera-se, portanto, que os investimentos efetuados representam um diferencial capaz de impactar positivamente o número de projetos em andamento até o quinto ano subsequente.

Os insumos (recursos) considerados foram somente os provenientes do Tesouro do Estado de São Paulo (Fonte 1). Portanto, assume-se que para o gestor público é relevante a eficiência com que foram empregados os recursos públicos do Tesouro colocados à disposição das unidades da APTA. Se algumas unidades são mais bem sucedidas em captar outros recursos, através dos fundos especiais de despesas e de projetos com suporte financeiro da União, de estados ou municípios e da iniciativa privada, cabe a esses financiadores analisar a eficiência com que suas verbas foram empregadas.

Como os insumos foram agregados através de um índice - portanto, envolvendo quocientes -, o modelo DEA de retornos variáveis à escala (BCC) é o mais adequado, conforme demonstraram Hollingsworth e Smith (2003).

Cooper, Seiford e Tone (2007) destacam que uma regra básica do modelo envoltório é que o número de DMUs seja igual ou superior ao máximo entre o número de produtos multiplicado pelo número de insumos, e a soma de produtos e insumos multiplicada por três. Portanto, como estão disponíveis sete observações por ano (as unidades da APTA), os modelos foram ajustados com um produto (geração ou transferência de conhecimento) e um insumo (a soma de despesas com pessoal, custeio e investimentos).

Em seguida, foi empregada a estratégia utilizada por Arcelus e Arocena (2000), de construção de uma fronteira intertemporal, que consiste em considerar as observações temporais como diferentes observações de uma série seccional. Dessa forma, com o número de observações atingindo 70, foi possível ajustar modelo com dois produtos (geração e transferência) e três insumos (despesas com pessoal, custeio e investimentos).

Por último, foi efetuada a análise em janelas, que permite que o desempenho de uma unidade seja comparado ao dela mesmo em outros 
anos, e com os de outras unidades no mesmo período (RAMANATHAN, 2003). As janelas guardam analogia com a tradicional análise de médias móveis em série de tempo. Como o PPA é definido por períodos de quatro anos, optou-se por efetuar essa análise com dados de quadriênios, substituindo as observações de dois anos em cada conjunto, totalizando quatro painéis de dados para o período analisado (2004-2013).

Atualmente, a execução de diversas atividades dos institutos de pesquisa depende da captação de outras fontes, devido à escassez de recursos do Tesouro. Por isso, comparou-se a eficiência relativa das unidades da APTA nesse quesito, tomando-se como produto os valores captados de agências de fomento, de outros órgãos governamentais e da iniciativa privada, somados aos recursos obtidos através dos fundos especiais de despesa (FEDs). Um modelo DEA intertemporal foi utilizado, tomando-se como produto os recursos de outras fontes empregados no ano em curso, conforme informações existentes no Simpa, e como insumos os recursos do Tesouro em custeio e em investimentos, além do número de pesquisadores de cada unidade. Como parte dos recursos utilizados em determinado ano foi captada em anos anteriores - e, portanto, dependeram dos insumos disponíveis naquela época -, os insumos foram inseridos no modelo com defasagens: além de to(ano em curso), também em $\mathrm{t}-1$, $\mathrm{t}-2$, e $\mathrm{t}-3$, resultando em uma especificação com um produto e 12 insumos.

\section{Resultados e discussão}

As médias geométricas dos índices de eficiência na geração de conhecimento (pesquisas em andamento),oriundos das fronteiras ajustadas ano a ano, mostram o IZ em primeiro lugar, com índice igual a 1. Isso significa que o IZ esteve determinando as fronteiras de eficiência das unidades da APTA em todos os dez anos de indicadores do PPA analisados. Em seguida apareceu o IAC, com média dos índices de eficiência na geração de conhecimento atingindo 0,95 e que esteve sobre as fronteiras nos cinco primeiros anos da série (2004 a 2008)e novamente em 2011. A participação do IAC no total de pesquisas decresceu, passando de 50,6\% em 2004 para 28,8\% em 2013, ao contrário da do IZ, que aumentou de 5,1\% em 2004 para 8,2\% em 2013 (Tabela 1).

Ainda com média de índices acima de 0,8 igual a 0,83 - surgiu o IB, que esteve na fronteira de geração de conhecimento em 2011 e cujas pesquisas em andamento representaram $13,4 \%$ do total da APTA em 2004, passando para 16,6\% em 2013. O DDD (Apta Regional) também se situou ao redor da marca de $80 \%$ de eficiência, com média de índices atingindo 0,79 e fazendo parte das fronteiras de eficiência nos seis últimos anos da série, reflexo do crescimento de sua participação no total de pesquisas da Apta, que evoluiu de 17,6\% em 2004 para 33,1\% em 2013.

Revista Brasileira de Monitoramento e Avaliação | Número 9 | Janeiro-Junho de 2015 
- TABELA 1: ÍNDICES DE EFICIÊNCIA TÉCNICA NA GERAÇÃO E TRANSFERÊNCIA DE CONHECIMENTO E NA CAPTAÇÃO DE RECURSOS INSTITUTOS DE PESOUUISA VINCULADOS À APTA, 2004 A 2013

\begin{tabular}{|c|c|c|c|c|}
\hline \multirow[b]{2}{*}{ UNIDADE } & \multicolumn{2}{|c|}{ CORTESSECIONAIS (1) } & \multicolumn{2}{|c|}{ INTERTEMPORAL (2) } \\
\hline & GERAÇÃO & TRANSFERÊNCIA & $\begin{array}{c}\text { GERAÇÃO E } \\
\text { TRANSFERÊNCIA }\end{array}$ & $\begin{array}{l}\text { CAPTAÇÃO DE } \\
\text { RECURSOS }\end{array}$ \\
\hline IAC & 0,9452 & 0,7064 & 0,8556 & 0,9368 \\
\hline IB & 0,8311 & 0,9854 & 0,9588 & 0,2301 \\
\hline Ital & 0,7236 & 0,9861 & 0,8388 & 0,4137 \\
\hline IEA & 0,5488 & 0,8967 & 0,9486 & 0,3314 \\
\hline Pesca & 0,4635 & 0,2975 & 0,3929 & 0,4801 \\
\hline IZ & 1 & 0,6906 & 0,6697 & 0,5276 \\
\hline DDD (Apta Regional) & 0,7932 & 0,6584 & 0,7475 & 0,1882 \\
\hline
\end{tabular}

(1) Médias geométricas de índices calculados ano a ano.

(2) Médias geométricas de índices calculados com painel de dados do período.

Fonte: Dados básicos do Simpa/PPA, SIGEO, SIGA e unidades da APTA.

Com média de índices de eficiência na geração de conhecimento de 0,72, o Ital fez parte da fronteira de eficiência em 2011, ano de sua máxima participação nas pesquisas em andamento na APTA (10,1\%), que aumentou desde 2004 (4,7\%), apesar de ter caído nos dois últimos anos, fixando-se em 8,4\% em 2013. O IEA e o Pesca ficaram com as menores médias de índices de eficiência na geração de conhecimento, 0,55 e 0,46, respectivamente. $\mathrm{O}$ IEA tangenciou as fronteiras de eficiência no triênio 2009-2011 (índices da ordem de $0,99)$, enquanto que o Pesca atingiu a maior marca no início da série $(0,84)$.

Projetando-se todas as unidades para as fronteiras de eficiência estimadas ano a ano - ou seja, caso todas as unidades estivessem operando nos níveis de máxima eficiência -, os resultados dos modelos sugerem que os índi- ces de geração de conhecimento (pesquisas em andamento) no período 2004-2013 poderiam ser $21,1 \%$ maiores, mantida a mesma dotação de recursos.

O Ital e o IB, com médias ao redor de 0,99, lideraram os índices de eficiência na transferência de conhecimento. Em nove dos dez anos analisados, o Ital ficou sobre a fronteira de eficiência, e o IB, em oito desses anos. Logo a seguir apareceu o IEA, com índice médio de 0,90, situando-se na fronteira em seis anos da série. Esses três institutos destacaram-se em diferentes componentes do índice de transferência de conhecimento: o Ital respondeu por percentuais entre $23,3 \%$ a $55,6 \%$ do total de pessoas treinadas; o IB por $45,5 \%$ a $63,0 \%$ das análises laboratoriais; o IEA por $12,8 \%$ a $25,1 \%$ dos acessos aos sites institucionais da APTA. O IAC (índice médio de 0,71), 
o IZ (índice médio igual a 0,69) e o DDD (APTA Regional, com índice médio de 0,66) também estiveram na fronteira de eficiência de transferência de conhecimento em algum momento na série estudada: os primeiros em dois anos, e o terceiro, no ano de 2006. Apenas o Instituto de Pesca deixou de fazer parte dessa fronteira no período 2004-2013. Deve-se destacar que um dos indicadores do Pesca, as visitas ao museu e ao aquário mantidos por esse instituto - que em alguns meses superaram 11 mil -, não são consideradas no PPA, uma vez que os usuários não são, necessariamente, relacionados ao agronegócio. Isso também acontece com as visitas ao Planeta Inseto, mantido pelo IB, que superaram a marca de 60 mil em 2012.

Se todas as unidades estivessem operando nos níveis de máxima eficiência, mantendo-se inalterados os recursos utilizados, os índices de transferência de conhecimento seriam 31,8\% maiores no período 2004-2013.

Os resultados da fronteira intertemporal - em que geração e transferência foram consideradas como produtos separadamente, assim como os três insumos (custeio, investimentos e despesas com pessoal)-também revelaram que nenhuma das unidades obteve coeficiente igual a 1 nos dez anos considerados. Ou seja, todos se situaram abaixo da fronteira em pelo menos um ano. Quatro institutos mantiveram médias de índices acima de 0,8: IB, IEA, IAC e Ital, os dois primeiros com níveis de eficiência acima de 90\%. O DDD apareceu com índice médio acima de 0,7, e o IZ, próximo dessa marca. O Pesca aparece em patamar inferior, provavelmente devido à subestimação de seus esforços na transferência de conhecimento, conforme citado anteriormente.

Considerando-se que todas as observações estivessem sobre a fronteira de eficiência, chegar-se-ia a aumentos de $26,5 \%$ e $24,8 \%$, respectivamente, nos indicadores de geração e de transferência de conhecimento das unidades da APTA, com os recursos disponíveis no período 2004-2013.

No item eficiência na captação de recursos - no PPA, representada pelos recursos de outras fontes (exceto Fonte 1, Tesouro do Estado) utilizados no ano corrente - percebe-se uma grande diferença entre os níveis de eficiência do IAC (índice igual a 0,94) e das demais unidades da APTA. Até o ano de 2008, o Ital também se situava na fronteira de captação, mas a partir de 2009 foi ultrapassado pelo IZ e pelo Pesca nesse indicador.

No primeiro dos quatro painéis de dados definidos para a análise em janelas, o quadriênio 2004-2007, seis das sete unidades da APTA estiveram sobre a fronteira de eficiência na geração e transferência de conhecimento em pelo menos um ano. A única exceção foi o DDD. No quadriênio seguinte, 2006-2009, cinco unidades frequentaram a fronteira de eficiência, com exceção novamente do DDD, e desta vez também do Pesca (Tabela 2).

Revista Brasileira de Monitoramento e Avaliação | Número 9 | Janeiro-Junho de 2015 
TABELA 2: ANÁLISE EM JANELAS DE ÍNDICES DE EFICIÊNCIA TÉCNICA NA GERAÇÃO E TRANSFERÊNCIA DE CONHECIMENTO -INSTITUTOS DE PESOQUISA VINCULADOS À APTA, 2004 A 2013(1)

\begin{tabular}{|c|c|c|c|c|c|c|c|c|c|c|}
\hline UNIDADE & 2004 & 2005 & 2006 & 2007 & & & & & & \\
\hline IAC & 1 & 1 & 1 & 1 & & & & & & \\
\hline IB & 0,9150 & 1 & 0,8868 & 1 & & & & & & \\
\hline Ital & 0,6480 & 1 & 1 & 0,9535 & & & & & & \\
\hline IEA & 1 & 1 & 1 & 1 & & & & & & \\
\hline Pesca & 1 & 0,4700 & 0,5885 & 0,4759 & & & & & & \\
\hline IZ & 1 & 0,9252 & 0,6584 & 0,8969 & & & & & & \\
\hline DDD & 0,4634 & 0,6063 & 0,8188 & 0,8603 & & & & & & \\
\hline UNIDADE & & & 2006 & 2007 & 2008 & 2009 & & & & \\
\hline IAC & & & 1 & 1 & 0,8431 & 0,8147 & & & & \\
\hline IB & & & 0,9354 & 1 & 0,8201 & 1 & & & & \\
\hline Ital & & & 1 & 0,9535 & 0,9142 & 0,6952 & & & & \\
\hline IEA & & & 1 & 1 & 0,6736 & 1 & & & & \\
\hline Pesca & & & 0,7391 & 0,5747 & 0,3777 & 0,3281 & & & & \\
\hline IZ & & & 1 & 1 & 0,5993 & 0,4744 & & & & \\
\hline DDD & & & 0,7978 & 0,8167 & 0,7427 & 0,8701 & & & & \\
\hline UNIDADE & & & & & 2008 & 2009 & 2010 & 2011 & & \\
\hline IAC & & & & & 1 & 1 & 0,9993 & 0,8100 & & \\
\hline IB & & & & & 1 & 1 & 1 & 1 & & \\
\hline Ital & & & & & 1 & 0,9339 & 1 & 1 & & \\
\hline IEA & & & & & 1 & 1 & 1 & 1 & & \\
\hline Pesca & & & & & 0,5978 & 0,4892 & 0,5078 & 0,4017 & & \\
\hline IZ & & & & & 1 & 0,7349 & 1 & 1 & & \\
\hline DDD & & & & & 0,9559 & 1 & 1 & 0,8299 & & \\
\hline UNIDADE & & & & & & & 2010 & 2011 & 2012 & 2013 \\
\hline$I A C$ & & & & & & & 1 & 0,8366 & 0,7845 & 0,9574 \\
\hline IB & & & & & & & 1 & 1 & 1 & 1 \\
\hline Ital & & & & & & & 1 & 1 & 0,7494 & 1 \\
\hline IEA & & & & & & & 1 & 1 & 0,9670 & 1 \\
\hline Pesca & & & & & & & 0,4091 & 0,3952 & 0,2764 & 0,3269 \\
\hline IZ & & & & & & & 1 & 1 & 0,9525 & 1 \\
\hline DDD & & & & & & & 1 & 0,8343 & 0,9510 & 1 \\
\hline
\end{tabular}

(1) As cores de preenchimento das caselas indicam as regiōes da fronteira próximas às quais as unidades estão operando: \#na de retornos crescentes; na de decrescentes; e branco, na região de retornos constantes à escala. Fonte: Dados básicos do Simpa/PPA, SIGEO, SIGA e unidades da APTA. 
Já nos dois quadriênios seguintes (20082011 e 2010-2013), apenas o Pesca não determinou a fronteira de eficiência, enquanto as outras seis unidades alcançaram índice igual a $1 \mathrm{em}$ pelo menos um dos anos. Reitere-se que os indicadores de transferência de conhecimento não contemplam as visitas ao museu e ao aquário mantidos por aquele instituto, que contribuem também para a difusão da imagem institucional da APTA.

Da tabela de resultados da análise em janelas, observa-se que um percentual elevado de observações $(37,5 \%)$ operava sob retornos crescentes à escala, em sua quase totalidade referentes ao IZ, Pesca, IEA e Ital, que são unidades menores. Isso significa que esses institutos estiveram com recursos aquém de seus níveis ótimos. Esse fato é ainda mais evidente nos resultados da análise intertemporal, em que 35 observações (50\% do total) ficaram na região de retornos crescentes à escala. Dessa forma, a aplicação de recursos adicionais teria resultado em aumentos mais do que proporcionais nos resultados, ao menos naqueles medidos pelos indicadores de geração e transferência de conhecimento utilizados no PPA.

Projetando-se todas as unidades da APTA para as fronteiras de eficiência das diferentes janelas analisadas, os modelos ajustados indicam aumentos potenciais de $15,8 \%$ na geração de conhecimento e de $16,2 \%$ em sua transferência, fixando-se os recursos empregados no período 2004-2013.

Na adaptação do modelo de quadrantes da pesquisa científica de Stokes (2005), utilizada por Rio (2009), considerando os índices de eficiência para as atividades de geração e transferência do conhecimento calculados ano a ano, observa-se que seis das sete unidades da APTA colapsam no ponto de máximo do quadrante de Pasteur. Isso significa que essas unidades estiveram nas fronteiras de geração e de transferência de conhecimento em pelo menos um dos anos do período 2004-2013. O ponto de máximo do Pesca (IPmáx), também localizado no quadrante de Pasteur, mostrou nível elevado do índice de geração de conhecimento e nível mais modesto do índice de transferência, provavelmente associado aos motivos citados anteriormente (Figura 1).

Os pontos médios dos índices de seis institutos - IAC,IB, Ital, IEA, IZ e DDD - também ficaram no quadrante de Pasteur, mostrando que na maioria dos anos do período 20042013 os indicadores do PPA dessas unidades apontam para esforços de gestão dirigidos tanto à geração quanto à transferência de conhecimento. No caso do Pesca (IP), cuja média tangenciou o quadrante de Bohr, o resultado dos indicadores ilustra a predominância de esforços na geração de conhecimento antes comentada.

Revista Brasileira de Monitoramento e Avaliação | Número 9 | Janeiro-Junho de 2015 
Os pontos mínimos dos índices foram mais heterogêneos. No caso do IB (IBmín), ainda localizado no quadrante de Pasteur, demonstra equilíbrio nas atividades de geração e transferência de conhecimento. O IAC (IACmín) e o IZ (IZmín) tiveram pontos mínimos ainda com bons resultados na geração de conhecimento, portanto, localizados no quadrante de Bohr. Resultado oposto foi revelado pelo ponto mí- nimo do Ital (ITALmín), no qual a transferência de conhecimento permaneceu em nível elevado. Os pontos de mínimo do IEA (IEAmín), Pesca (IPmín) e DDD (DDDmín), por outro lado, indicam que essas unidades experimentaram anos, durante o período 2004-2013, em que tiveram dificuldade em manter elevados os índices, tanto de geração, quanto de transferência de conhecimento.

- FIGURA 1: DISTRIBUIÇÃO dOS ÍNDICES DE EFICIÊNCIA NOS QUUADRANTES DA PESOLUISA CIENTÍFICA

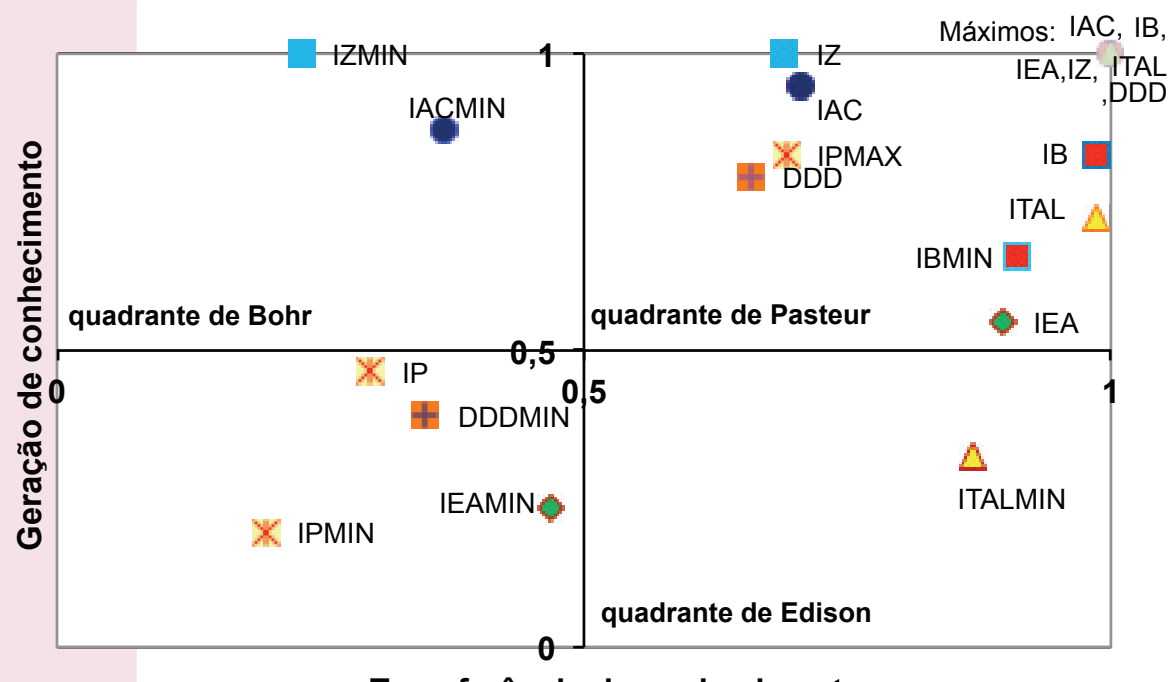

Transferência de conhecimento 


\section{Considerações finais}

Os resultados obtidos do ajuste dos modelos de eficiência revelaram que as unidades da APTA operaram nas fronteiras de eficiência da geração ou da transferência de conhecimento em algum ponto do período de 2004 a 2013.

A maioria das unidades apresentou médias anuais de índices de eficiência superiores a 0,7 , tanto para a geração de conhecimento, quanto para a transferência de conhecimento. Apenas uma unidade (IAC) destacou-se na captação de recursos.

Em fronteira intertemporal, considerando-se simultaneamente a geração e a transferência de conhecimento, mais de $70 \%$ das unidades exibiram índices médios superiores a 0,7.

Os resultados dos modelos sugerem que, se todas as unidades operassem em níveis máximos de eficiência, os índices de geração de conhecimento poderiam aumentar entre $15,8 \%$ e $26,5 \%$, e os de transferência de conhecimento, entre $16,2 \%$ e $31,8 \%$, mantendo-se inalterados os recursos utilizados.

A representação das médias dos índices de eficiência calculados anualmente, para a geração de conhecimento e para a transferência, em quadrantes de pesquisa, com seis das sete unidades situando-se no quadrante de
Pasteur, é um indicativo de comprometimento com a busca e difusão de conhecimento.

Para subsidiar os gestores dessas unidades, visando a aumentos de eficiência, extensões desse estudo podem utilizar indicadores mais desagregados, apontando, por exemplo, em quais dos componentes dos indicadores de transferência as unidades deveriam concentrar seus esforços. Os índices provenientes da análise em janelas permitem que o desempenho de cada unidade seja comparado aos de seus pares no mesmo período, e as causas de eventuais quedas de eficiência sejam clarificadas, explicitando-se se foram decorrentes da insuficiência ou da alocação de recursos.

Cabe destacar que os indicadores do PPA são limitados, principalmente para representar os resultados da geração de conhecimento nos institutos de pesquisa. Da mesma forma, revelaram-se ainda insuficientes para computar determinadas atividades relacionadas à transferência de conhecimento de algumas unidades, que alcançam grandes parcelas da sociedade.

\section{Agradecimentos}

Os autores agradecem a Sílvio de Barros Filho e Raquel Lopes pela coleta e organização de dados do Sistema de Informações Gerenciais da Execução Orçamentária (SIGEO) e do Sistema de Informações Gerenciais do Agronegócio (SIGA).

Revista Brasileira de Monitoramento e Avaliação | Número 9 | Janeiro-Junho de 2015 
ARCELUS, F. J.; AROCENA, P. Convergence and productive efficiency in fourteen OECD countries: a non-parametric frontier approach. International Journal of Production Economics, v. 66, n. 2, p. 105-117, jun. 2000.

BANKER, R. D.; CHARNES, A.; COOPER, W. W. Some models for estimating technical scale inefficiencies in data envelopment analysis. Management Science, v. 30, n. 9, p. 1078-1092, sep.1984.

CHARNES, A.; COOPER, W.; RHODES, E. Measuring the efficiency of decision making units. European Journal of Operational Research, v. 2, n. 6, p. 429-444, 1978.

COELLI, T. A Guide to DEAP Version 2.1: a Data Envelopment Analysis (Computer) Program. Armidale: University of New England/Department of Econometrics/Centre for Efficiency and Productivity Analysis, 1996. (CEPA Working Paper 96/08).

COELLI, T. J. et al. An Introduction to efficiency and productivity analysis. 2nd ed. New York: Springer, 2005.
COOPER, W. W.; SEIFORD, L. M.; TONE, K. Data Envelopment Analysis: a comprehensive text with models, applications, references and DEA-Solver software. 2nd ed. New York: Springer, 2007.

FØRSUND, F. R.; LOVELL, C. A. K.; SCHMIDT, P. A survey of frontier productions functions and of their relationship to efficiency measurement. Journal of Econometrics, v. 13, n. 1, p. 5-25, may. 1980.

GONTIJO, V. Instrumentos de Planejamento e Orçamento. Brasília: Câmara dos Deputados. Disponível em: <http://www2.camara.leg.br/ atividade-legislativa/ orcamentobrasil/cidadao/entenda/cursopo/planejamento.html>. Acesso em: set. 2014.

HOLLINGSWORTH, B.; SMITH, P. The use of ratios in data envelopment analysis. Applied Economics Letters, v.10, n.11, p. 733-735, 2003.

KHAN, A. S.; SILVA, L. M. Capital social das comunidades beneficiadas pelo Programa de Combate à Pobreza Rural - PCPR/Projeto São José - PSJ - estado do Ceará. Revista de Economia e Sociologia Rural, v. 43, n.1, p. 101-117, jan./ mar. 2005. 
RAMANATHAN, R. An Introduction to Data Envelopment Analysis: a tool for performance measurement. New Delhi: Sage Publications, 2003.

RAO, D. S. P.; COELLI, T. J. Economic Growth, Productivity Change and Inequality: methodology for the assessment of economic performance of nations. Armidale: University of New England/Department of Econometrics/Centre for Efficiency and Productivity Analysis, aug. 1999.

RIO, C. T. Análise do modelo de gestão da Agência Paulista de Tecnologia dos Agronegócios (APTA). Campinas: IG/UNICAMP, 2009. (Dissertação de Mestrado).

SACHS, J. A Riqueza de Todos: a construção de uma economia sustentável em um planeta superpovoado, poluído e pobre. Rio de Janeiro: Nova Fronteira, 2008.

SEIFORD, L. M.; THRALL, R. M. Recent developments in DEA: the mathematical programming approach to frontier analysis. Journal of Econometrics, v. 46, n. 1/2, p. 7-38, oct./Nov, 1990.
STOKES, D. E. 0 Quadrante de Pasteur: a ciência básica e a inovação tecnológica. Campinas, Editora Unicamp, 2005.

VEIGA FILHO, A.; VICENTE, J. R; MARTINS, R. Eficiência na geração e transferência do conhecimento: uma análise dos Polos Regionais de Desenvolvimento Tecnológico dos Agronegócios do Estado de São Paulo. In: SIMPÓSIO BRASILIERO DE PESOUUISA OPERACIONAL, 43.,2011, Ubatuba. Anais... Rio de Janeiro: SOBRAPO, 2011. CD-ROM.

VICENTE, J. R. Determinantes da adoção de tecnologia na agricultura paulista. Estudos Econômicos, v. 28, n.3, p. 421-451, set./dez. 1998.

VICENTE, J. R. Pesquisa, Adoção de Tecnologia e Eficiência na Produção Agrícola. São Paulo: APTA, 2002. (Série Discussão APTA 2).

VICENTE, J. R; MARTINS, R. Eficiência na geração e transferência de tecnologia: uma análise de Institutos de Pesquisa Agropecuária do Estado de São Paulo. In: SIMPÓSIO DE GESTÃO DA INOVAÇÃO TECNOLÓGICA, 24.,2006, Gramado. Anais... Gramado: ANPAD, 2006. CD-ROM.

Revista Brasileira de Monitoramento e Avaliação | Número 9 | Janeiro-Junho de 2015 